\title{
A New hybrid generalized CG- method for non-linear functions
}

Abbas Y. Al-Bayati

profabbasalbayati@yahoo.com

College of Computc
Hamsa Th. Chilmerane

hamsathrot@uomosul.edu.iq University of Mosul

Received on: 6/4/2005

Accepted on: 9/10/2005

\section{ABSTRACT}

In this paper a new extended generalized conjugate gradient algorithm is proposed for unconstrained optimization, which is considered as anew inverse hyperbolic model.In order to improve the rate of convergence of the new technique, a new hybrid technique between the standard F/R CGmethod and Sloboda CG-method using quadratic and non-quadratic models is proposed by using exact and inexact line searches. This method is more efficient and robust when applied on number of well-known nonlinear test function.

Keywords: F/R, exact line search, inexact line search.

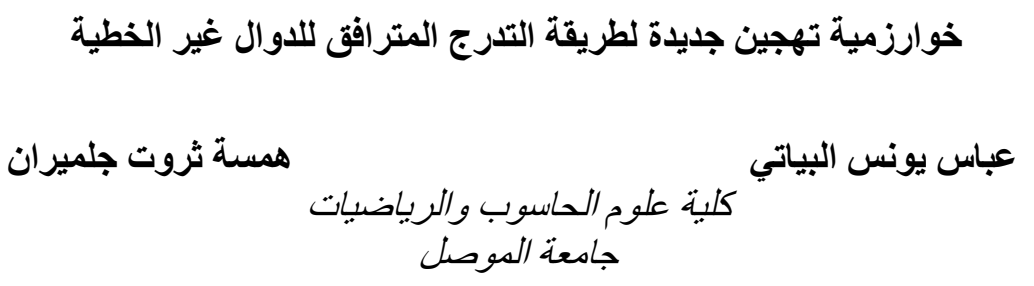

تاريخ قبول البحث: 2005/10/9

تاريخ استلام البحث: 2005/4/6

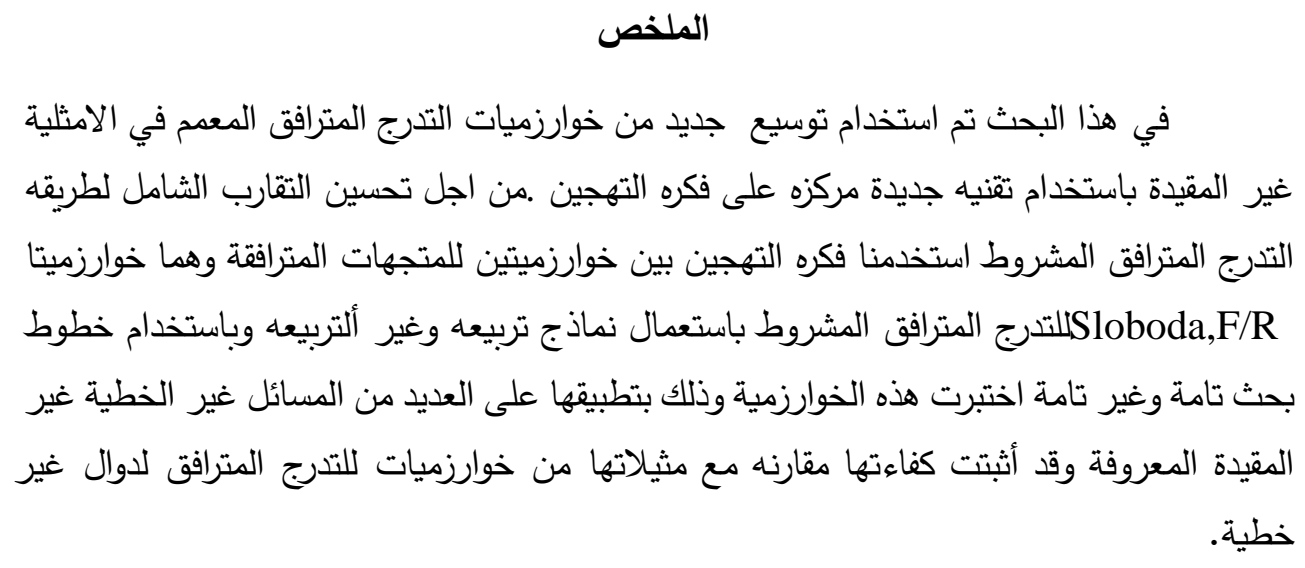




$$
\text { الكلمات المفتاحية: F/R، خط بحث تام، خط بحث غير تام. }
$$

\section{Introduction}

The conjugate gradient method is particularly useful for minimizing function of mult variables because it does not require the storage of any matrices. However, the rate of convergence of the algorithm is only linear unless the iterative procedure is "restarted" occasionally .At present it is usual to restart every $n$ or $(n+1)$ iterations, where $n$ is the number of variables, but it is known that frequency of restarts should depend on the objective function.

$$
d_{k+1}=\left[\begin{array}{lrr}
-g_{k} & \text { for } & k=1 \\
-g_{k+1}+\beta_{k} d_{k} & & \text { fork } \succ 1,
\end{array}\right]
$$

CG method is useful to find minimum of a function $f: R^{n} \longrightarrow R$. In general, the method has the following form

$$
x_{k+1}=x_{k}+\lambda_{k} d_{k}
$$

where $g_{k}$ denotes the gradient $\nabla f\left(x_{k}\right), d_{k}$ is the search direction, $\lambda_{k}$ is a steplength obtained by a line search, and $\beta_{k}$ is chosen so that it

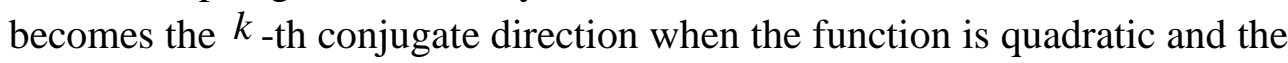
line search is exact, a well known formula for $\beta_{k}$ is given by

$$
\begin{aligned}
& \beta_{k}=\frac{y_{k}^{T} g_{k+1}}{d_{k}^{T} y_{k}} \quad(\text { Hestenes \& Stiefel , 1952) } \\
& \beta_{k}=\frac{\left\|g_{k+1}\right\|^{2}}{\left\|g_{k}\right\|^{2}}(\text { Fletcher \& Reeves, 1964) } \\
& \beta_{k}=\frac{g_{k+1}^{T}\left(g_{k+1}-g_{k}\right)}{\left\|g_{k}\right\|^{2}} \quad \text { ( Polak \& Ribire, 1969) } \\
& \beta_{k}=\frac{-\left\|g_{k+1}\right\|^{2}}{d_{k}^{T} g_{k}} \quad(\text { Dixon, } 1975) \\
& \beta_{k}=\frac{-g_{k+1}^{T} y_{k}}{d_{k}^{T} g_{k}} \quad \text { (Al-Bayati \& Al-Assady, 1986) }
\end{aligned}
$$

where $y_{k}=g_{k+1}-g_{k}$

The successive directions are conjugate vectors for the successive 
gradients obtained as the method progresses.

\section{Non Quadratic Models:}

The conjugate gradient methods so far discussed is a local quadratic representation of the objective function .In problems when the quadratic representation is not good, or when we are remote from such a region, quadratic function $F(q(x))$, where $F$ is monotonic increasing ,may be better to represent the objective and thus it gives an advantage to algorithm based on this model. In order to obtain better global rate of convergence for minimization algorithms when applied to more general functions than the quadratic. In this paper several new algorithms, which are invariant to nonlinear scaling of quadratic functions are proposed. There is some precedent for this approach, if $q(x)$ is quadratic function then a function $f$ is defined as nonlinear scaling of $q(x)$ if the following condition holds

$$
f=F(q(x)) \quad \frac{d f}{d q}=F^{\prime}>0 \quad \text { and } \quad q(x)>0
$$

where $x^{*}$ is minima of $q(x)$ with respect to $x$.

The following properties are immediately derived from the above conditions.

i - every contour line of $q(x)$ is a contour line of $f$;

ii - if $x^{*}$ is minimizer of $q(x)$ then it is a minimizer of $f$;

iii - that $x^{*}$ is global minimum of $q(x)$ does not necessarily mean that it is

a global minimum of $f$. For more details see Boland et al., (1979).

Many authors have proposed special models as follows:

$$
\begin{aligned}
& F(q(x))=(q(x))^{\mathrm{p}} \quad \mathrm{p} \succ 0 \quad \text { Fried (1971) } \\
& F(q(x))=\in_{1} q(x)+\frac{1}{2} \in_{2} q^{2}(x) \quad \in_{1}, \in_{2} \text { scaler } \quad \text { Boland et al., (1979) } \\
& F(q(x))=\frac{\epsilon_{1} q(x)}{1-\in_{2} q(x)} \quad \in_{2} \prec 0 \quad \text { Al-Bayati (1993) } \\
& F(q(x))=(\log (\in q(x))-1) \quad \in \succ 0 \text { Al-Bayati (1995) } \\
& F(q(x))=\log \left(\frac{\epsilon_{1} q(x)}{\in_{2} q(x)+1}\right) \quad \in_{2} \prec 0 \quad \text { Al-Assady and Huda (1997) } \\
& F(q(x))=\sin (\in q(x))
\end{aligned}
$$

3. New Extended Generalized Conjugate Gradient Method by using Hyperbolic Inverse Model:

Let 
$f_{k}=\sinh ^{-1} q_{k}$

Then

$f_{k}=\ln \left(q_{k}+\sqrt{1+q_{k}^{2}}\right)$

$e^{f_{k}}=q_{k}+\sqrt{1+q_{k}^{2}}$

$e^{f_{k}}-q_{k}=\sqrt{1+q_{k}^{2}}$

$e^{2 f_{k}}-2 e^{f_{k}} q_{k}+q_{k}^{2}=1+q_{k}^{2}$

$e^{2 f_{k}}-1=2 e^{f_{k}} q_{k}$

$q_{k}=\frac{e^{2 f_{k}}-1}{2 e^{f_{k}}}$

$q_{k}=\frac{e^{f_{k}}-e^{-f_{k}}}{2}$

Since $\rho_{k}$ is a parameter, which is defined as

$\rho_{k}=f_{k}^{\prime} / f_{k+1}^{\prime}$

$\rho_{k}=\frac{\frac{1}{\sqrt{1+q_{k}^{2}}}}{\frac{1}{\sqrt{1+q_{k+1}^{2}}}}$

$\rho_{k}=\frac{1}{\sqrt{1+\left(\frac{e^{f_{k}}-e^{-f_{k}}}{2}\right)^{2}}} / \frac{1}{\sqrt{1+\left(\frac{e^{f_{k+1}}-e^{-f_{k+1}}}{2}\right)^{2}}}$

\section{Sloboda CG-Method:}

The rate of convergence of a variety of CG-algorithm has been investigated by many authors: the most general results have been given by Baptist and Storey (1977) where it was also shown that the algorithms with ELS (exact line searches) have the property of $n$-step quadratic convergence (Store, 1977). In order to improve the rate of convergence of CG-algorithm it is necessary to construct special algorithms for more general function than the quadratic .In series of papers: Fried (1971); Boland et al., (1979); Tassopoulous and Storey (1984);AL-Bayati et al. (1994),(1995) various algorithms have been suggested which are efficient for special nonquadratic models. Sloboda (1980) first developed an algorithm which generates conjugate direction with inexact line searches and has the same 
rate of convergence as the classical CG-method without an error vector.

\subsection{Outlines of Sloboda CG-Method:}

Step1: $x_{0} \in R^{n}$, initial point

Step 2: set $k=1, \mathrm{~g}_{1}^{-}=-\mathrm{g}_{1}$ and $\mathrm{d}_{1}=\mathrm{g}_{1}^{-}$

Step 3: set $x_{k+1}=x_{k}+\lambda_{k} d_{k}$

Step 4: compute $g_{k+1}^{+}=g_{k+1}(x-\lambda d / 2)$

Step 5: test for convergence: if achieved stop, if not continue

Step 6: if $k=n$ or (any equivalent restarting criterion) go to step 3 else continue.

Step 7: compute $g_{k+1}^{-}=\omega g_{k+1}^{+}-g_{k}^{-}$where $\omega=d_{k+1}^{T} g_{k+1}^{-} / g_{k+1}^{T} g_{k+1}^{+}$

Step 8: $d_{k+1}=-g_{k+1}^{-}+\beta_{k} d_{k}$ where $\beta=g_{k+1}^{-T} g_{k+1}^{-T} / g_{k}^{T} g_{k}$

Step 9: set $k=k+1$ and go to Step 3 .

\section{Hybrid Conjugate Gradient Methods:}

Despite the numerical superiority of Polak-Riebiere (P/R) algorithm over Fletcher-Reeves (F/R) algorithm, the later has better theoretical properties than the former. Under certain conditions $\mathrm{F} / \mathrm{R}$-method can be shown to have global convergence with exact line search (Powell, 1986) and also with inexact line search satisfying the strong Wolfe-Powell condition. (see Al-Baali, 1985).

Normally this leads to speculation on the best way to choose $\beta_{k}$. Touati-Ahmed and Storey (1995) proposed the following hybrid algorithm:

Step1: If $\hat{\zeta}\left\|g_{k+1}\right\|^{2} \leq(2 \zeta)^{k+1}$, with ${ }^{\frac{1}{2}}>\zeta>\xi$ and $\hat{\zeta}>0$, go to step 3 .

Otherwise, set $\beta_{k}=0$.

Step2: If $\beta_{k}^{P / R}<0$ set $\beta_{k}=\beta_{k}^{F / R}$. Otherwise go to step 3 .

Step3: If $\beta_{k}^{P / R} \leq\left(\frac{1}{2} \zeta\right)\left\|g_{k+1}\right\|^{2} /\left\|g_{k}\right\|_{\text {with }}^{2} \zeta>\xi$, set $\beta_{k}=\beta_{k}^{F / R}$.

Otherwise set $\beta_{k}=\beta_{k}^{P / R}$.

Here $^{\xi}, \zeta$ and $\hat{\zeta}$ are user supplied parameters. This hybrid was shown to be globally convergent under both exact and inexact line searches and to be quite competitive with P/R-algorithm and F/R-algorithm. See Hu and Storey ( 1991 ) .

Touati and Storey suggested also the following algorithm to compute 
the conjugancy coefficient $\beta_{k}$ :

Step1: If $\beta_{k}^{P / R}<0$, then $\beta_{k}=\beta_{k}^{F / R}$, return to main program. Otherwise go to step2.

Step2: If $0 \leq g_{k+1}^{T} g_{k} \leq\left\|g_{k+1}\right\|^{2}$, then $\beta_{k}=\beta_{k}^{P / R}$; return to main program.

Otherwise, go to step3.

Step3: If

$$
\cos ^{2}\left(\theta_{k}\right) \geq \imath_{k}^{2} ; \text { where } \imath_{k}^{2}=\tau /\left[\left\|g_{k}\right\|^{2} \sum_{i=1}^{k}\left\|g_{i}\right\|^{-2}\right] \text { holds, }
$$

then $\beta_{k}=\beta_{k}^{P / R}$; return to main program. Otherwise, set

$\beta_{k}=\beta_{k}^{F / R}$; return to main program.

\section{New Suggestion for Hybrid CG-Methods:}

In this section we are going to study develop a new CG-method based on quadratic and non- quadratic models; taking the idea of exact and inexact line searches. The new technique use new hybrid idea between the standard F/R CG-algorithm and Sloboda (1980) CG-method.

\subsection{Outlines of the New Suggested Algorithm:}

Step1:Set $x_{0} \in R^{n}$,initial point

Step 2:Set $k=1$

Step3:Set $d_{k}=-g_{k}$

Step 4:Set $x_{k+1}=x_{k}+\lambda_{k} d_{k}$

Step 5:Check for convergence i.e, if $\left\|g_{k+1}\right\| \prec \varepsilon$, then stop else

Step 6: Find

$$
\rho_{k}=\frac{1}{\sqrt{1+\left(\frac{e^{f_{k}}-e^{-f_{k}}}{2}\right)^{2}}} / \frac{1}{\sqrt{1+\left(\frac{e^{f_{k+1}}-e^{-f_{k+1}}}{2}\right)^{2}}}
$$

Step 7: if $\frac{d_{k_{k}}^{T} g_{k+1}}{\left\|g_{k+1}\right\|}<0.2$ is satisfied go to step(8) otherwise go to step (13)

Step 8: $g_{k}=g\left(x_{k}\right)$

$$
g_{k+1}=g\left(x_{k+1}\right)
$$


Step 9:Find $\beta_{k}=\left\|g_{k+1}\right\|^{2} /\left\|g_{k}\right\|^{2}$

Step 10: check if $0 \prec \beta_{k} \prec 1$

Step 11: compute $d_{k+1}=-g_{k+1}+\beta_{k} d_{k}$, go to step 19 else

Step 12: compute $d_{k+1}=-g_{k+1}+\rho_{k} \beta_{k} d_{k}$, go to step 19

Step 13: find $g_{k+1}^{+}=g(x-\lambda d / 2)$

Step 14: compute $g_{k+1}^{-}=\varpi g^{+}{ }_{k+1}-g_{k}$, where $\varpi=d_{k+1}^{T} \bar{g}_{k+1} / g_{k+1}^{+} d_{k+1}^{T}$

Step15: Find $\beta_{k}=\left\|g^{-}{ }_{k+1}\right\|^{2} /\left\|g_{k}^{-}\right\|^{2}$

Step 16: check if $0 \prec \beta_{k} \prec 1$

Step 17: compute $d_{k+1}=-g^{-}{ }_{k+1}+\beta_{k} d_{k}$,go to step19 else

Step 18: compute $d_{k+1}=-g^{-}{ }_{k+1}+\rho_{k} \beta_{k} d_{k}$

Step 19:if $k=n$ or (any equivalent restarting critenion)go to step (2), else

Step 20: set, $k=k+1$ and go to step (3)

\section{Numerical Results:}

In order to assess the performance of the new proposed algorithm (Hybrid model). Three minimization algorithms are tested over (10) nonlinear unconstrained test functions with different dimensions see (Appendix).

All the results are obtained using (Pentium computer). All programs are written in FORTRAN language and for all cases the stopping criterion taken to be

$$
\left\|g_{k+1}\right\| \prec 1 \times 10^{-5}
$$

Algorithms in this chapter use ELS and ILS strategy which is the cubic fitting technique fully described in (Bunday, 1984).

The comparative performance for all of these algorithms are evaluated by considering NOF and NOI, where NOF is the number of function evaluations and NOI is the number of iterations.

The algorithms are:

1- Standard F/R CG-algorithm.

2- Sloboda CG-algorithm.

3- New Hybrid model algorithm. 
In Table (1) we represent comparison between new algorithm with Standard F/R CG-algorithm and Sloboda CG-algorithm.

Our numerical results, which are presented in Table (2) confirm that the Hybrid model algorithm is superior to both Standard CG-algorithm and Sloboda CG-algorithm with respect to the total number of NOF and NOI.

Table (1)

Comparison our new algorithm with standard F/R CG-algorithm and Sloboda CG-algorithm.

\begin{tabular}{|l|c|c|c|c|}
\hline Test fn. & Dim & $\begin{array}{c}\text { F/R CG } \\
\text { NOI(NOF) }\end{array}$ & $\begin{array}{c}\text { Sloboda CG } \\
\text { ALGORITHM } \\
\text { NOI(NOF) }\end{array}$ & $\begin{array}{c}\text { NEW } \\
\text { ALORITHM } \\
\text { NOI(NOF })\end{array}$ \\
\hline Powell & 4 & $79(193)$ & $60(150)$ & $50(107)$ \\
\hline Osp & 4 & $8(40)$ & $6(30)$ & $6(30)$ \\
\hline Cubic & 4 & $15(42)$ & $19(46)$ & $15(41)$ \\
\hline Shallow & 4 & $11(26)$ & $10(26)$ & $10(26)$ \\
\hline Cantreal & 4 & $40(269)$ & $38(209)$ & $37(246)$ \\
\hline Osp & 100 & $48(144)$ & $47(150)$ & $47(142)$ \\
\hline Beale & 100 & $57(118)$ & $69(142)$ & $62(128)$ \\
\hline Cubic & 100 & $49(106)$ & $67(184)$ & $17(41)$ \\
\hline Recip & 100 & $8(22)$ & $8(22)$ & $11(30)$ \\
\hline Strait & 100 & $9(20)$ & $12(26)$ & $11(24)$ \\
\hline Sum & 100 & $15(80)$ & $17(86)$ & $16(89)$ \\
\hline Strait & 500 & $9(20)$ & $12(26)$ & $15(32)$ \\
\hline Shallow & 500 & $20(46)$ & $18(43)$ & $21(46)$ \\
\hline
\end{tabular}




\begin{tabular}{|l|c|c|c|c|}
\hline Recip & 500 & $8(22)$ & $10(29)$ & $9(24)$ \\
\hline Sum & 500 & $26(144)$ & $25(136)$ & $22(112)$ \\
\hline \multicolumn{2}{|c|}{ Total } & $402(1292)$ & $418(1305)$ & $349(1118)$ \\
\hline
\end{tabular}

\section{REFERENCES}

[1] Al-Assady, N.H, and AL-Mashhdany, H. (1997) "A Rational Logarithmic Science Model for Unconstrained Non-Linear Optimization", Rafideen Science, J. Vol 8,No.2, pp. 107-17

[2] Al-Assady, N.H and Al-Ta'ai B.A. , (2002) "A New Non-Quadratic Model For Unconstrained Non-Linear Optimization" Journal of Al-Raffadin , Computer and Mathematical Science, No 48 , pp.150151.

[3] Al-Baali, M., (1985) "Descent Property and Global Convergence of the Fletcher-Reeves Method with Inexact Line Search" IMA, Journal of Numerical Analysis, Vol. 5, pp. 121-124.

[4] Al-Bayati, A.Y., (1993) "A New Non-Quadratic Model for Unconstrained Non-linear Optimization Method" Natural and applied series Mu'tah Journal for research and studies Mu'tah University, Jordan, Vol.8, No.1, pp. 133-155 .

[5] AL-Bayati, A.Y. and AL-Assady , N.H. (1994) A.Y, "Minimization of quadratic functions with inexact line search, journal of optimization Theory and Applications, Vol. 32, pp.139147.

[6] AL-Bayati, A.Y. and AL-Naemi , G.M., (1995),"A combined ECGV M method for nonlinear unconstrained optimization", Journal of Education and Science, Mosul University ,IRAQ, Vol.23, P.P. 207-214.

[7] Al-Bayati, A.Y. (1995) "A New Extended CG-Method for Nonlinear Optimization Method" Natural and applied series Mu'tah Journal for Research and Studies Mu'tah University, Jordan, Vol.10, No .6, pp. 69-87. 
[8] Baptist, P. and Store, J. (1977), "On the relation between quadratic termination and convergence properties of minimization algorithms, part 11, Numerical Mathematics" No.28, pp. 367-391.

[9] Boland W.R.;kamgnia,E.R.and Kowalik, J.S. ( 1979 ) "Extended CG-method with Restarts" journal of optimization theory and application, Vol. 28,pp.1-9 .

[10] Bundy, B. (1984) "Basic Optimization Method" Edward Arnold, Bedford Square, London, U.K.

[11] Dixon, L.C.W. (1975) "Conjugate Direction Without Linear Search" Journal of Institute Mathematics and it is Applications 11. PP: $317-328$.

[12] Fletcher, R. and Recves , (1964), "Function minimization by conjugate gradient" Compreter . J. 7, pp. 147 - 154.

[13] Fried, I.(1971) "N-step Conjugate Gradient Minimization Scheme for Non-Quadratic Function" AIAA jouranl, Vol. 19.pp.22862287.

[14] Hestenes, M.R. and Stiefel E. (1952) "Methods of Conjugate Gradients for Solving Linear Systems" Journal of Research of the National Burean of Standards, Vol. 49, pp:409-436.

[15] Hu,Y. and Storey, C., (1991) "Efficient Generalized Conjugate Gradient Algorithms", Part 1, Theory, Journal of Optimization Theory and Applications, Vol. 69, PP.129-137.

[16] Polak , E. (1969) "Computational Methods in Optimization" A Unified Approach, Academic Press, New York.

[17] Powell, M. J. D. (1986) "How Bad are the BFGS and DFP Method When the Objective Function in Quadratic" Math programming, Vol. 34, pp.34-47. London.

[18] Sloboda, F ( 1980 ) "A generalized conjugate gradient algorithm Numerical Mathematics" ,No. 43 pp. 223-230 .

[19] Store J. (1977) "On the relation between quadratic termination and convergence properties of minimization algorithms part $I$. Theory, Numerical Mathematics", Vol. 28, pp. 343-366. 


\section{Appendix}

1. Generalized Osp (Oren and Spedicato Function:

$$
f(x)=\left[\sum_{i=1}^{n} i x_{i}^{2}\right]^{2}
$$

$$
x_{0}=(1, \ldots)^{T}
$$

2. Generalized Cantreal Function:

$f(x)=\sum_{i=1}^{n / 4}\left[\begin{array}{l}\left(\exp \left(x_{4 i-3}\right)-x_{4 i-2}\right)^{4}+100\left(x_{4 i-2}-x_{4 i-1}\right)^{6}+ \\ \left.\arctan \left(x_{4 i-1}-x_{4 i}\right)\right)^{4}+x_{4 i-3}\end{array}\right]$,

$x_{0}=(1,2,2,2 ; \ldots)^{T}$

3. Generalized Recip Function:

$$
f(x)=\sum_{i=1}^{n / 3}\left[\left(x_{3 i-1}-5\right)^{2}+x_{9 i-1}^{2}+\frac{x_{3 i}^{2}}{\left(x_{3 i-1}-x_{3 i-2}\right)^{2}}\right], \quad x_{0}=(2,5,1 ; \ldots)^{T} \text {. }
$$

4.Generalized Powell Function:

$$
f(x)=\sum_{i=1}^{n / 4}\left[\begin{array}{l}
\left(x_{4 i-3}+10 x_{4 i-2}\right)^{2}+5\left(x_{4 i-1}-x_{4 i}\right)^{2}+ \\
\left(x_{4 i-2}-2 x_{4 i-1}\right)^{4}+10\left(x_{4 i-3}-x_{4 i}\right)^{4}
\end{array}\right],
$$

5. Generalized Cubic Function:

$$
\begin{array}{r}
x_{0}=(3,-1,0,3 ; \ldots)^{T} . \\
x_{0}=(-1.2,1, \ldots)^{T} .
\end{array}
$$

$f(x)=\sum_{i=1}^{n / 2}\left[100\left(x_{2 i}-x_{2 i-1}^{3}\right)^{2}+\left(1-x_{2 i-1}\right)^{2}\right]$,

$$
x_{0}=(-1.2,1, \ldots)^{T}
$$

6.Generalized Beale Function:

$$
\begin{aligned}
& f(x)=\sum_{i=1}^{n / 2}\left\{\begin{array}{l}
{\left[1.5-x_{2 i-1}\left(1-x_{2 i}\right)\right]^{2}+\left[2.25-x_{2 i-1}\left(1-x_{2 i}^{2}\right)\right]^{2}} \\
+\left[2.625-x_{2 i-1}\left(1-x_{2 i}^{2}\right]^{2}\right.
\end{array}\right\}, \\
& x_{0}=(-1,1, \ldots)^{T} .
\end{aligned}
$$

7.Generalized Shallow Function:

$$
\mathrm{f}(\mathrm{x})=\sum_{\mathrm{i}=1}^{\mathrm{n} / 2}\left[\mathrm{x}_{2 \mathrm{i}-1}^{2}-\mathrm{x}_{2 \mathrm{i}}\right]^{2}+\left(1-\mathrm{x}_{2 \mathrm{i}-1}\right)^{2}
$$




$$
x_{0}=(-2,-2 ; \ldots)^{T}
$$

8.Generalized Strait Function:

$f(\mathrm{x})=\sum_{i=1}^{n / 2}\left(x_{2 i-1}^{2}-x_{2 i}\right)^{2}+100\left(1-x_{2 i-1}\right)^{2}$,

9.Sum of Quatrics (SUM) function:

$$
x_{0}=(2,-2 ; \ldots)^{T} \text {. }
$$

$$
f(x)=\sum_{i=1}^{n}\left(x_{i}-i\right)^{4}
$$

$$
x_{0}=(1, \ldots)^{T}
$$

